\title{
A BLUE POINT SOURCE AT THE LOCATION OF SUPERNOVA 2011DH
}

\author{
Gastón Folatelli ${ }^{1}$, Melina C. Bersten ${ }^{1}$, Omar G. Benvenuto ${ }^{2,3}$, Schuyler D. Van Dyk $^{4}$, Hanindyo Kuncarayakti ${ }^{5,6}$, \\ KeIIChI Maeda ${ }^{7}$, TaKaya Nozawa ${ }^{8}$, Ken'IChi Nomoto ${ }^{1,9}$, Mario HamuY $^{5,6}$, and Robert M. Quimby ${ }^{1}$ \\ ${ }^{1}$ Kavli Institute for the Physics and Mathematics of the Universe (WPI), The University of Tokyo, Kashiwa, Chiba 277-8583, Japan; gaston.folatelli@ipmu.jp \\ ${ }^{2}$ Facultad de Ciencias Astronómicas y Geofísicas, Universidad Nacional de La Plata, Paseo del Bosque S/N, B1900FWA La Plata, Argentina \\ ${ }^{3}$ Instituto de Astrofísica de La Plata (IALP), CONICET, Argentina \\ ${ }^{4}$ Spitzer Science Center/Caltech, Mailcode 220-6, Pasadena, CA 91125, USA \\ ${ }^{5}$ Millennium Institute of Astrophysics (MAS), Casilla 36-D, Santiago, Chile \\ ${ }^{6}$ Departamento de Astronomía, Universidad de Chile, Casilla 36-D, Santiago, Chile \\ ${ }^{7}$ Department of Astronomy, Kyoto University, Kitashirakawa-Oiwake-cho, Sakyo-ku, Kyoto 606-8502, Japan \\ ${ }^{8}$ National Astronomical Observatory of Japan, Mitaka, Tokyo 181-8588, Japan \\ Received 2014 August 15; accepted 2014 August 28; published 2014 September 10
}

\begin{abstract}
We present Hubble Space Telescope (HST) observations of the field of the Type IIb supernova (SN) 2011dh in M51 performed at $\approx 1161$ rest-frame days after explosion using the Wide Field Camera 3 and near-UV filters $F 225 \mathrm{~W}$ and $F 336 \mathrm{~W}$. A star-like object is detected in both bands and the photometry indicates it has negative $(F 225 W-F 336 W)$ color. The observed object is compatible with the companion of the now-vanished yellow supergiant progenitor predicted in interacting binary models. We consider it unlikely that the SN is undergoing strong interaction and thus estimate that it makes a small contribution to the observed flux. The possibilities of having detected an unresolved light echo or an unrelated object are briefly discussed and judged unlikely. Adopting a possible range of extinction by dust, we constrain parameters of the proposed binary system. In particular, the efficiency of mass accretion onto the binary companion must be below 50\%, if no significant extinction is produced by newly formed dust. Further multiband observations are required in order to confirm the identification of the object as the companion star. If confirmed, the companion star would already be dominant in the UV-optical regime, so it would readily provide a unique opportunity to perform a detailed study of its properties.
\end{abstract}

Key words: binaries: close - supernovae: general - supernovae: individual (SN 2011dh)

Online-only material: color figures

\section{INTRODUCTION}

After decades of sustained progress in stellar evolution theory, the connection between different types of core-collapse supernovae $(\mathrm{SNe})$ and their progenitors remains partly unknown. Hydrogen-rich, Type II SNe, have been found to arise from red supergiant stars, as expected from evolutionary models (Smartt 2009; Van Dyk 2012). Conversely, the origin of hydrogen-poor $\mathrm{SNe}$ (Types Ib, Ic, and IIb) is unclear and one important question is how their progenitors expel the hydrogen envelope. This process may be regulated by wind, which is expected to be stronger for larger progenitor mass. However, there is compelling evidence that binarity and, in particular, mass-transfer processes in interacting binaries, must play an important role in the evolution of most massive stars (Sana et al. 2012).

In this context, characterizing the progenitors of Type IIb $\mathrm{SNe}$, which retain a small fraction of their hydrogen envelopes, becomes particularly relevant. One of the best-studied objects of this type is SN 1993J, whose progenitor has been identified as a massive binary system composed of a K-type supergiant progenitor plus a B-type supergiant companion (Maund et al. 2004; Maund \& Smartt 2009; Fox et al. 2014). More recently, the discovery of the Type IIb SN 2011dh in M51 provided the opportunity - with improved observational capability —of identifying yet another progenitor of this relatively uncommon type of core-collapse SNe. Very early light curves and spectra were interpreted by Arcavi et al. (2011), using an analytical representation of the post-shock-breakout cooling, to be indicative of

\footnotetext{
9 Hamamatsu Professor.
}

a compact progenitor, possibly a Wolf-Rayet (WR) star. However, through inspection of deep pre-explosion images from the Hubble Space Telescope (HST), Maund et al. (2011) and Van Dyk et al. (2011) independently found an object at the SN location that was compatible with an extended yellow supergiant star (YSG), and not with a WR star. The question arose as to whether this object was the actual progenitor, a companion in a binary system, or an unrelated object in the line of sight. While Van Dyk et al. (2011) speculated that the actual progenitor was an unseen hot compact star, Maund et al. (2011) suggested the possibility of having a YSG progenitor in a close binary system, similar to the case of SN 1993J. By increasing the mass-loss rates in single stellar evolution models, Georgy (2012) was able to produce YSG progenitors of core-collapse $\mathrm{SNe}$, although without providing a physical motivation for the modified mass loss. Soon after, Soderberg et al. (2012) suggested the progenitor must have been a compact star based on early radio observations of the SN. The latter was subsequently supported by Krauss et al. (2012) and Bietenholz et al. (2012). However, Maeda (2012) and Horesh et al. (2013) later questioned the ability of radio data to indicate the actual progenitor size, thus arguing against the necessity of a compact progenitor.

In the meantime, Bersten et al. (2012) performed a more detailed, numerical hydrodynamical modeling of the SN light curve and expansion velocity, and showed that the progenitor could not have been a massive WR star and, very importantly, that it had a radius of $R \gtrsim 200 R_{\odot}$, compatible with a YSG star. Bersten et al. (and later Benvenuto et al. 2013, in greater detail) presented evolutionary models of close binary systems 

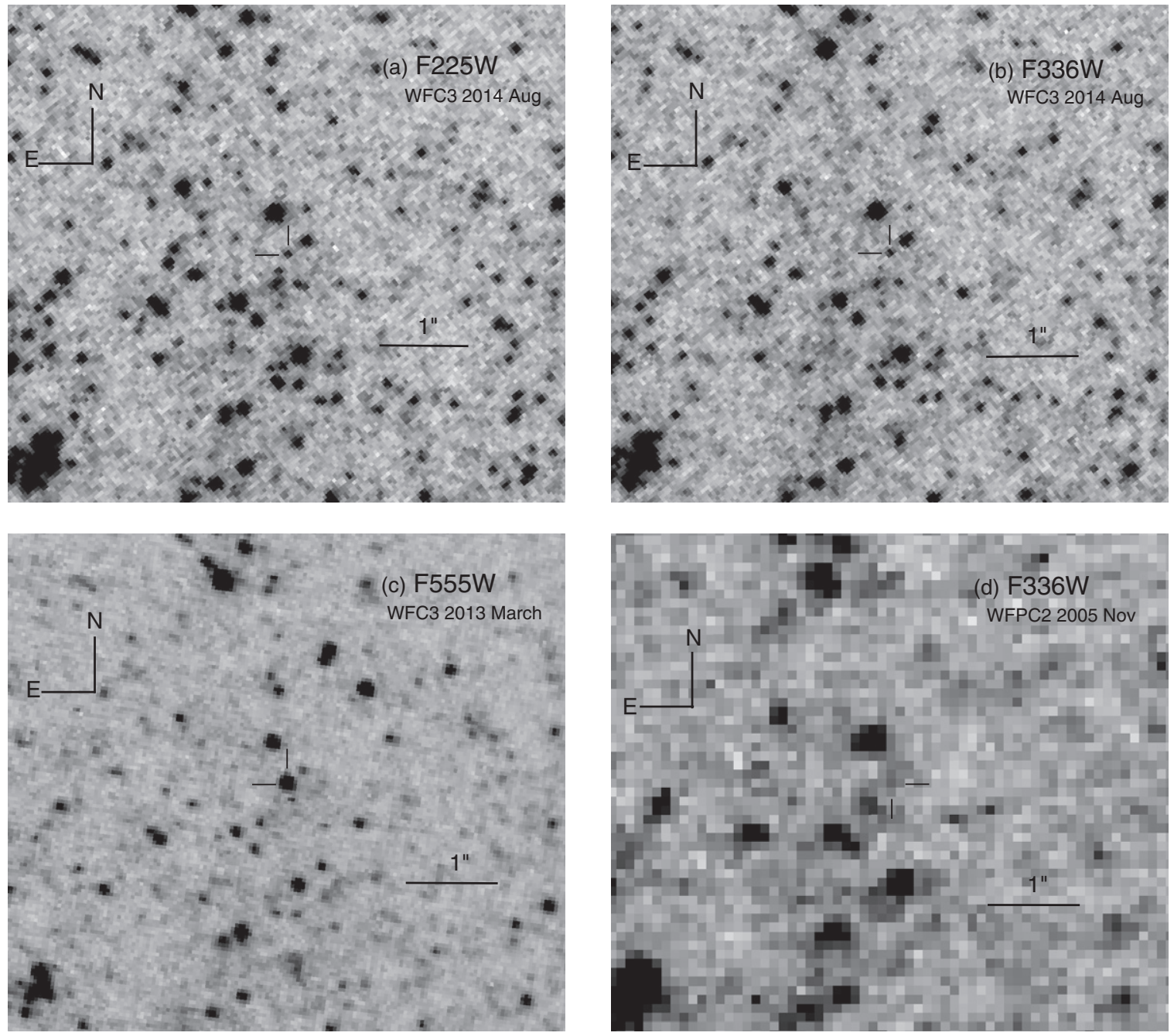

Figure 1. (a) and (b) Portions of the deep F225W- and F336W-band images obtained with the HST and WFC3/UVIS on 2014 August 7.2 UT. (c) 2013 March image obtained with WFC3/UVIS and filter $F 555 \mathrm{~W}$ showing the fading SN ejecta (Van Dyk et al. 2013). (d) Pre-explosion image obtained with the WFPC2 on 2005 November showing the progenitor detection (Maund et al. 2011; Van Dyk et al. 2011). Images in all four panels are centered at the SN location and scale and orientation are indicated. A point source is detected in both near-UV images from 2014 August, as indicated with tick marks. The position of the source is coincident with that of the SN in the 2013 March image to a projected distance of $\lesssim 0.15 \mathrm{pc}$.

that naturally provided a complete picture of the progenitor of SN 2011dh. Their calculations were able to simultaneously explain the explosion of a YSG star with a final mass compatible with the result of the hydrodynamical modeling, with a small amount of hydrogen in the envelope, suitable for producing a $\mathrm{SN}$ IIb, and with a companion star that would not be detected in the pre-explosion images.

The debate was finally settled by Van Dyk et al. (2013) with new HST optical imaging of the SN site obtained 21 months after explosion (this was confirmed by Ergon et al. 2014a) using ground-based images. The new images showed the preexplosion object had disappeared, thus proving that the YSG star had exploded. At the moment of those observations the SN ejecta were too bright to determine the presence of the companion star proposed by Bersten et al. (2012) and Benvenuto et al. (2013). It is only recently that it is possible to find that last piece of the puzzle that can confirm the binary nature of the progenitor. Observations should be carried out in the UV range to improve the chances of detecting the hot companion.

This Letter presents deep near-UV imaging of the site of SN 2011dh obtained with HST when the SN had an age of about 1161 days. The observations were used to search for the companion star of the YSG progenitor and to characterize the properties of the progenitor system.

\section{OBSERVATIONS AND PHOTOMETRY}

The site of SN 2011dh was re-observed on 2014 August 7.2 $\mathrm{UT}^{10}$ with HST and the Wide Field Camera 3 (WFC3) using the UVIS channel and filters $F 225 W$ and $F 336 W$. The observations were obtained through Cycle 21 program GO-13426 (PI: J. Maund) with which our program GO-13433 (PI: G. Folatelli) was merged once it was realized that both programs shared the same target, similar science, and similar instrumental setup. The exposure times were $3772 \mathrm{~s}$ in $F 225 \mathrm{~W}$, and $1784 \mathrm{~s}$ in $F 336 \mathrm{~W}$. In Figure 1 we show the images around the location of the SN. For comparison, we show a previous image obtained with WFC3/UVIS and filter $F 555 \mathrm{~W}$ on 2013 March 2 through program GO-13029 (PI: A. V. Filippenko). This 2013 image clearly shows the fading SN, which was also detected in a $F 814 W$-band image obtained simultaneously (Van Dyk et al. 2013). Figure 1 also shows the pre-explosion

\footnotetext{
$\overline{10}$ Dates are given in UT time throughout the paper.
} 
$F 336 W$-image obtained with the Wide Field Planetary Camera 2 (WFPC2) on 2005 November 13 (GO-10501; PI: Chandar), where the progenitor was detected with low signal-to-noise ratio (Van Dyk et al. 2011; Maund et al. 2011).

A point source is identified at the location of the $\mathrm{SN}$ in both 2014 August images (Folatelli et al. 2014). We performed image registration of our $2014 F 336 \mathrm{~W}$ image relative to the $F 555 \mathrm{~W}$ image from 2013, using 35 common stars. The formal rms uncertainty in the geometric transformation was 0.08 pixel in each direction. The transformed location of the $\mathrm{SN}$ was offset from the blue object by only 0.05 pixel in each axis. Adding both sources of uncertainty in quadrature, the new object is coincident with the $\mathrm{SN}$ to 0.1 pixel, or 4 mas. At an assumed distance to M51 of 7.8 Mpc (Ergon et al. 2014a, from an average of several measurements), the new object is within $\approx 0.15 \mathrm{pc}$ from the $\mathrm{SN}$ position. We performed point-spread function (PSF) photometry on the HST images using the Dolphot v2.0 package (Dolphin 2000). The resulting VEGAMAG magnitudes for the detected object are $m_{\mathrm{F} 225 \mathrm{~W}}=24.57 \pm 0.11 \mathrm{mag}$, and $m_{\mathrm{F} 336 \mathrm{~W}}=24.94 \pm 0.11 \mathrm{mag}$.

\section{ANALYSIS}

\subsection{Nature of the Detected Object}

The object detected in our new HST images coincides with the SN location, as shown in Section 2. We thus tested whether the detected flux could be explained by the fading $\mathrm{SN}$ alone or if it required an additional source. We estimated the contribution from the SN ejecta at an age of 1161 days based on previous observations of SN 2011dh, and assuming the light-curve decline followed that of SN 1987A at a similar age. SN 1987A provides the richest available data set of latetime UV observations. Even if its early-time evolution was different from that of SN 2011dh, at the phase considered here, it is fair to assume the light curves of both $\mathrm{SNe}$ are regulated by radioactive decay processes and thus show a similar shape. We thus assumed there is no strong interaction of the ejecta with the circumstellar medium (CSM). In the following we conservatively assumed only Galactic extinction of $A_{V}=0.10 \mathrm{mag}$ for SN 2011dh (Schlafly \& Finkbeiner 2011), and a total extinction of $A_{V}=0.53 \pm 0.06 \mathrm{mag}$ for SN 1987A (see Pun et al. 1995). A standard reddening law of Cardelli et al. (1989) was adopted, with $R_{V}=3.1$.

The procedure for estimating the UV flux of the $\mathrm{SN}$ is depicted in Figure 2. We matched the $B$-band light curve of SN 1987A from Hamuy \& Suntzeff (1990) and Walker \& Suntzeff (1991) to the latest photometry of SN $2011 \mathrm{dh}$ at $\approx 700$ days from Ergon et al. (2014b). From this we determined an offset of $11.5 \mathrm{mag}$ between both $\mathrm{SNe}$. Assuming the difference in extinction given above, the offset applied in the $U$ band was of 10.8 mag. We then calculated synthetic photometry in the $U, B, F 225 \mathrm{~W}$, and $F 336 \mathrm{~W}$ bands using the spectra of SN 1987A published by Pun et al. (1995), after correcting for the difference in reddening. The synthetic photometry was roughly consistent with the observed $U$ - and $B$-band photometry, which provides confidence for its use to derive $F 225 W$ and $F 336 W$ magnitudes. The spectra of SN 1987A extended until about 800 days after explosion. There is a final spectrum obtained at about 1050 days, but it is too noisy in the near-UV range to be used here.

We extrapolated the synthetic photometry in $F 225 \mathrm{~W}$ and $F 336 \mathrm{~W}$, to the epoch of our HST observations. For this purpose, we used a cubic polynomial fit to the $U$-band light curve of SN 1987A between 700 and 1500 days, and assumed the

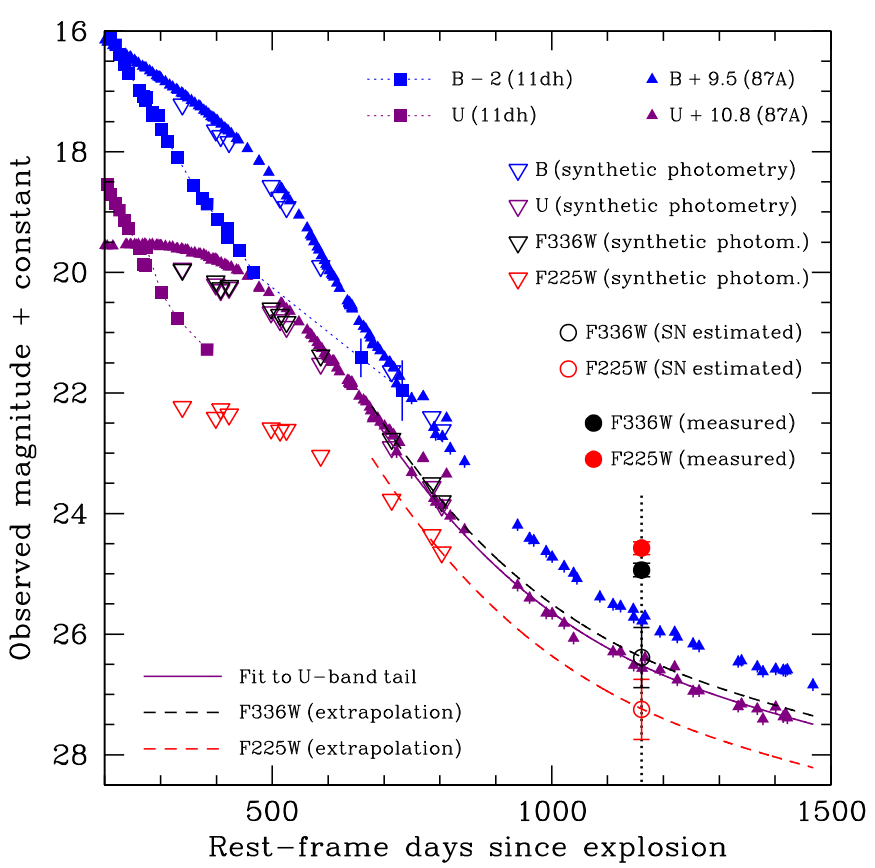

Figure 2. Estimated brightness of the fading SN. Squares indicate the $U$ - and $B$-band light curves of SN 2011dh from Ergon et al. (2014b). Filled triangles show the light curves of SN 1987A (Hamuy \& Suntzeff 1990; Walker \& Suntzeff 1991) shifted to match the evolution of SN 2011dh (see the text). Open triangles show the synthetic photometry in the HST bands computed from spectra of SN 1987A (Pun et al. 1995) and shifted to the scale of SN 2011dh. The solid curve is a fit to the late-time decline of the $U$-band light curve of SN 1987A. Dashed curves are the same fit, shifted vertically to match the synthetic light curves in $F 225 W$ and $F 336 W$ bands. The vertical dotted line indicates the epoch of our HST observations where the SN brightness was estimated from the shifted decline fits.

(A color version of this figure is available in the online journal.)

$F 225 W$ and $F 336 W$ bands followed the same decline rate. With this, the extrapolated magnitudes of the SN ejecta in the $H S T$ bands were $m_{\mathrm{F} 225 \mathrm{~W}}(1161$ days $)=27.3 \pm 0.5 \mathrm{mag}$, and $m_{\mathrm{F} 336 \mathrm{~W}}(1161$ days $)=26.4 \pm 0.5 \mathrm{mag}$. The uncertainties were estimated based on the extinction uncertainty, and on the variation in ( $U$-near-UV) colors among the latest spectra of SN 1987A. This analysis suggests that in the absence of strong CSM interaction, the $\mathrm{SN}$ was $\approx 2.7 \mathrm{mag}$ and $\approx 1.5 \mathrm{mag}$ fainter than the observed object in $F 225 W$ and $F 336 W$, respectively.

The emission from the expanding $\mathrm{SN}$ ejecta may be revitalized if it encounters substantial circumstellar material and a shock is produced. This would cause a flattening of the light curve and it would introduce signatures in the spectrum. No evidence of strong interaction was seen in the optical light curves or spectra of SN 2011dh at 700 days after explosion (Shivvers et al. 2013; Ergon et al. 2014b; Jerkstrand et al. 2014), in X-rays at $\approx 500$ days (Maeda et al. 2014), or in radio at $\approx 1000$ days (A. Kamble 2014, private communication). Contrary to SN 1993J which showed signs of interaction in this wavelength range as soon as 100 days after explosion (e.g., Filippenko et al. 1994; Kohmura et al. 1994), SN 2011dh appears to be comparatively free of CSM (Maeda et al. 2014). Even if CSM interaction strengthened after $\approx 1000$ days, it would be difficult to explain the observed blue UV color. Spectra of SN 1993J at similar ages (Fransson et al. 2005) show no strong lines in the range of the $F 225 \mathrm{~W}$ filter that could be responsible for the observed color.

An alternative explanation to the observed flux may be an unresolved echo of the SN light reflected in our direction. Any 


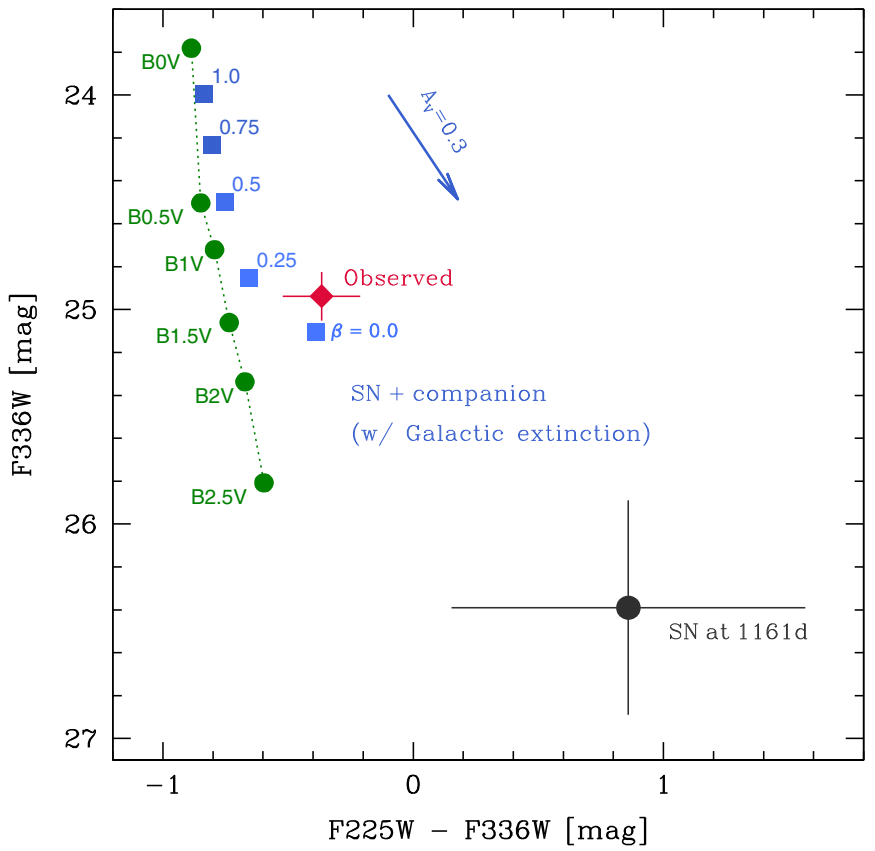

Figure 3. $F 336 W$ vs. $(F 225 W-F 336 W)$ color-magnitude diagram. The observed object is shown with a red diamond, without correcting for extinction. The black dot shows the expected location of the SN, as estimated in Figure 2. Blue squares indicate the expected observations from the $\mathrm{SN}$ plus a binary companion (SN + companion) according to the models of Benvenuto et al. (2013), for varying mass-accretion efficiency ( $\beta$; labeled next to each point). A distance of 7.8 Mpc was adopted, and the SN + companion model photometry was reddened by Galactic extinction of $A_{V}=0.10$ mag to reproduce the observations. Green dots show the location of main sequence stars for the same distance and reddening. The arrow indicates the reddening vector for an additional extinction of $A_{V}=0.3 \mathrm{mag}$ that may be produced by dust in M51 (see the text for details).

(A color version of this figure is available in the online journal.)

such echo should arise from within $\sim 1$ pc from the SN. At that distance, we only expect to find CSM created from the pre-SN mass loss, which can be assumed to have too low a density to produce a detectable echo. In addition, light from the SN should be backward-scattered, which is relatively inefficient in the near-UV (Sugerman 2003).

It is in principle possible that the observed emission is due to an unrelated source in the line of sight. However, we consider this unlikely because such an object, presumably an OB type star, should be projected at $\lesssim 0.15$ pc from the SN, which leaves a small volume across the disk of the face-on galaxy M51.

\subsection{Putative Companion Star Properties}

From the analysis in the previous section it is likely that the detected object is the companion of the YSG progenitor of SN 2011dh. If this is the case, its observed near-UV color and magnitude can be used to characterize the companion star, as shown in Figure 3. Adopting a Galactic extinction only and an average distance of $7.8 \pm 0.9 \mathrm{Mpc}$, the absolute magnitudes of the object are $M_{\mathrm{F} 225 \mathrm{~W}}=-5.11 \pm 0.29 \mathrm{mag}$, and $M_{\mathrm{F} 336 \mathrm{~W}}=-4.66 \pm 0.29 \mathrm{mag}$. Extinction by dust in M51 is uncertain. Arcavi et al. (2011) and Ritchey \& Wallerstein (2012) used the strength of Na I D lines in the SN spectra to suggest that extinction was low. However, from color analysis of the stellar population in the vicinity of the SN, Murphy et al. (2011) found extinction of $A_{V} \approx 0.3 \mathrm{mag}$. Assuming the latter value, the corrected absolute magnitudes would be $M_{\mathrm{F} 225 \mathrm{~W}}=-5.88 \mathrm{mag}$ and $M_{\mathrm{F} 336 \mathrm{~W}}=-5.15 \mathrm{mag}$. The allowed range of absolute magnitudes is roughly compatible with main-sequence, B0-B2 type stars.

There is the possibility that additional dust is formed in the SN ejecta, which would introduce extra extinction. Nozawa et al. (2010) predict that significant amounts of dust can form in the ejecta of Type IIb SNe approximately between 300 and 700 days after the explosion, but that the average grain size is smaller than in Type II P SNe. In addition, UV emission from a putative hot star present within the ejecta can destroy the small dust grains. Dust formation has been suggested for SN 2011dh as early as 150 days (Sahu et al. 2013; Ergon et al. 2014b; Jerkstrand et al. 2014), however, it is difficult to quantify the amount of extinction in the near-UV regime. If present, additional dust would imply an intrinsically more luminous and bluer object than what was derived above.

Figure 3 also shows the HST photometry compared with the predictions from the close-binary evolutionary models of Benvenuto et al. (2013). The suggested progenitor system had zero-age masses of $16 M_{\odot}$ (the exploding star) and $10 M_{\odot}$ (the accreting companion), and an initial orbital period of 125 days. Depending on the value of the mass-accretion efficiency parameter, $\beta$, the model predicted different final masses, luminosities, and temperatures for the companion. The companion star spectra were adopted from atmosphere models of Kurucz (1993) with an effective temperature and surface gravity as given by the binary models and were scaled to a distance of M51. To this we added an SN contribution using the spectrum of SN $1987 \mathrm{~A}$ at $\approx 800$ days, dereddened, and scaled to the expected at 1161 days as estimated in Section 3.1 (Figure 2). The resulting "SN + companion" spectra were reddened by Galactic extinction and synthetic photometry was computed in the $F 225 \mathrm{~W}$ and $F 336 W$ bands. As shown in Figure 3, the observed object is remarkably compatible with the $\mathrm{SN}+$ companion photometry provided the accretion efficiency is low. For comparison, the diagram shows the estimated position of the SN ejecta. Assuming additional extinction in the host galaxy of $A_{V} \lesssim 0.3 \mathrm{mag}$, the proposed companion star is compatible with the observations for $\beta \lesssim 0.5$. This implies a mass range of $10 \lesssim M \lesssim 16 M_{\odot}$, for the secondary star at the time of the SN explosion. Note, however, that we expect a certain degree of degeneracy in the models between $\beta$ and the initial mass of the companion, which would require more extensive observations and modeling to disentangle the degeneracy. If $\beta$ is small, one can expect part of the mass lost by the primary to be in the surroundings and to eventually be shocked by the ejecta. In the models of Benvenuto et al. (2013), however, the mass-transfer rate is high $\left(\sim 10^{-3} M_{\odot} \mathrm{yr}^{-1}\right)$ only at $\sim 10^{5}$ yr before the explosion, so we can assume the ejecta (expanding at $\sim 10^{4} \mathrm{~km} \mathrm{~s}^{-1}$ ) will reach this material (if expelled at $\sim 10 \mathrm{~km} \mathrm{~s}^{-1}$ ) not earlier than $\sim 10^{2} \mathrm{yr}$.

The predicted $\mathrm{SN}+$ companion photometry for the optical HST observations obtained on 2014 August 10 (GO-13426) are $25.8 \lesssim m_{\mathrm{F} 435 \mathrm{~W}} \lesssim 26.6 \mathrm{mag}, 26.0 \lesssim m_{\mathrm{F} 555 \mathrm{~W}} \lesssim 26.7 \mathrm{mag}$, and $26.0 \lesssim m_{\mathrm{F} 814 \mathrm{~W}} \lesssim 26.4 \mathrm{mag}$. If there is additional dust formed in the ejecta, the object would be intrinsically brighter, which would imply a larger companion mass, presumably due to a larger accretion efficiency.

\section{CONCLUSIONS}

We have shown the detection in deep near-UV images obtained with HST of a blue point source at the location of the Type IIb SN 2011dh. The source's photometry is compatible with it being the progenitor companion predicted by Bersten 
et al. (2012) and Benvenuto et al. (2013). We consider it unlikely that the observed flux is due to the SN ejecta itself, to a light echo, or to an unrelated object in the line of sight. SN 2011dh would thus be the second core-collapse SN, after SN 1993J (Maund et al. 2004; Fox et al. 2014), to show strong evidence of a binary companion to the progenitor. If confirmed, the companion of SN $2011 \mathrm{dh}$ would already be the dominant source in the optical-UV regime, thus providing a unique opportunity for analyzing its properties.

These observations may provide important clues about the evolutionary paths of massive stars and the role of binarity in the envelope removal among $\mathrm{H}$-poor SNe. The fact that the two best-studied Type IIb SNe most probably had binary progenitors is particularly suggestive of a dominant binary channel for this subtype of $\mathrm{SNe}$, especially considering the difficulty of single stellar evolution models to produce progenitors that lose most but not all of their H-rich envelopes.

Our analysis allowed us to provide a range of valid massaccretion efficiency $\beta \lesssim 0.5$ for the specific models presented by Benvenuto et al. (2013). In general, this quantity is included in the models as a free, unconstrained parameter. However, a conclusive derivation of the binary model properties would require a more detailed study and further observational data covering a wider wavelength range. At the same time, such observations may allow us to better determine the degree of interaction between the ejecta and the CSM, the possible contamination from a light echo, and the amount of extinction from pre-existing and newly formed dust.

We thank Armin Rest for his light-echo calculations and discussion. This research is supported by the World Premier International Research Center Initiative (WPI Initiative), MEXT, Japan, and by Grants-in-Aid for Scientific Research $(23224004,23540262,23740141,23740175,26400222$, 26400223, 26800100). M.H. and H.K. acknowledge support from the Millennium Institute of Astrophysics (MAS; Programa Iniciativa Científica Milenio del Ministerio de Economía, Fomento y Turismo de Chile, grant IC120009). H.K. is supported by FONDECYT (grant 3140563). O.G.B. is member of the Carrera del Investigador Científico of CIC, Argentina.

\section{REFERENCES}

Arcavi, I., Gal-Yam, A., Yaron, O., et al. 2011, ApJL, 742, L18

Benvenuto, O. G., Bersten, M. C., \& Nomoto, K. 2013, ApJ, 762, 74

Bersten, M. C., Benvenuto, O. G., Nomoto, K., et al. 2012, ApJ, 757, 31

Bietenholz, M. F., Brunthaler, A., Soderberg, A. M., et al. 2012, ApJ, 751,125

Cardelli, J. A., Clayton, G. C., \& Mathis, J. S. 1989, ApJ, 345, 245

Dolphin, A. E. 2000, PASP, 112, 1383

Ergon, M., Jerkstrand, A., Sollerman, J., et al. 2014, arXiv:1408.0731

Ergon, M., Sollerman, J., Fraser, M., et al. 2014, A\&A, 562, A17

Filippenko, A. V., Matheson, T., \& Barth, A. J. 1994, AJ, 108, 2220

Folatelli, G., Van Dyk, S. D., Benvenuto, O. G., et al. 2014, ATel, 6375, 1

Fox, O. D., Bostroem, K. A., Van Dyk, S. D., et al. 2014, ApJ, 790, 17

Fransson, C., Challis, P. M., Chevalier, R. A., et al. 2005, ApJ, 622, 991

Georgy, C. 2012, A\&A, 538, L8

Hamuy, M., \& Suntzeff, N. B. 1990, AJ, 99, 1146

Horesh, A., Stockdale, C., Fox, D. B., et al. 2013, MNRAS, 436, 1258

Jerkstrand, A., Ergon, M., Smartt, S. J., et al. 2014, arXiv:1408.0732

Kohmura, Y., Inoue, H., Aoki, T., et al. 1994, PASJ, 46, L157

Krauss, M. I., Soderberg, A. M., Chomiuk, L., et al. 2012, ApJL, 750, L40

Kurucz, R. 1993, ATLAS9 Stellar Atmosphere Program and $2 \mathrm{~km} / \mathrm{s}$ Grid, Kurucz CD-ROM (Cambridge, MA: Smithsonian Astrophysical Observatory), 13

Maeda, K. 2012, ApJ, 758, 81

Maeda, K., Katsuda, S., Bamba, A., Terada, Y., \& Fukuzawa, Y. 2014, ApJ, 785,95

Maund, J. R., Fraser, M., Ergon, M., et al. 2011, ApJL, 739, L37

Maund, J. R., \& Smartt, S. J. 2009, Sci, 324, 486

Maund, J. R., Smartt, S. J., Kudritzki, R. P., Podsiadlowski, P., \& Gilmore, G. F. 2004, Natur, 427, 129

Murphy, J. W., Jennings, Z., Williams, B., Dalcanton, J. J., \& Dolphin, A. E. 2011, ApJL, 742, L4

Nozawa, T., Kozasa, T., Tominaga, N., et al. 2010, ApJ, 713, 356

Pun, C. S. J., Kirshner, R. P., Sonneborn, G., et al. 1995, ApJS, 99, 223

Ritchey, A. M., \& Wallerstein, G. 2012, ApJL, 748, L11

Sahu, D. K., Anupama, G. C., \& Chakradhari, N. K. 2013, MNRAS, 433, 2

Sana, H., de Mink, S. E., de Koter, A., et al. 2012, Sci, 337, 444

Schlafly, E. F., \& Finkbeiner, D. P. 2011, ApJ, 737, 103

Shivvers, I., Mazzali, P., Silverman, J. M., et al. 2013, MNRAS, 436, 3614

Smartt, S. J. 2009, ARA\&A, 47, 63

Soderberg, A. M., Margutti, R., Zauderer, B. A., et al. 2012, ApJ, 752, 78

Sugerman, B. E. K. 2003, AJ, 126, 1939

Van Dyk, S. D., Li, W., Cenko, S. B., et al. 2011, ApJL, 741, L28

Van Dyk, S. D. 2012, in IAU Symp. 279, Death of Massive Stars: Supernovae and Gamma-Ray Bursts, ed. P. Roming, N. Kawai, \& E. Pian (Cambridge: Cambridge Univ. Press), 110

Van Dyk, S. D., Zheng, W., Clubb, K. I., et al. 2013, ApJL, 772, L32

Walker, A. R., \& Suntzeff, N. B. 1991, PASP, 103, 958 